Marx, Karl

1975. Early Writings. Quinton Hoare, ed. Rodney Livingstone and Gregor Benton, trans. New York: Penguin Books.

\title{
Uncommon Ground: Cultural Landscapes and Environmental Values (1997), by Veronica Strang, Oxford ; New York : Berg, 1997. xiv, 309 pp.
}

\section{Reviewed by Shelley Greer, School of Anthropology \& Archaeology, James Cook University, Townsville, Queensland Australia.}

In this work, Strang examines concepts of "landscape" and "environment", comparing the perspectives of Aboriginal people and pastoralists living in the Mitchell River catchment of Cape York Peninsula. She does this by referring to the history of this part of Cape York, the "landscapes of the past"; by describing the contemporary cultural landscapes of both groups and the way in which they describe and organize space, and by undertaking a comparison of the values invested in land through education, representations and cosmology.

The concept of comparing the values of these two groups is extremely interesting and timely, given the current debate in Australia surrounding Native Title. Strang's study is all the more relevant as the geographic area she has chosen for her study borders the traditional lands of the Wik people whose Native Title claim has served, after Mabo, as the test case for Native Title through both the legal and political systems.

Strang makes several important points. First, she convincingly makes the point that concepts such as "landscape" and "environment" are culturally constructed and that this obviously affects the "value" of these. In this regard, Strang's findings resonate with other work in Cape York that critiques the notion of 'heritage' in terms of indigenous perspectives of landscape (S. Greer 1995). Perspectives such as these are particularly important in areas like Cape York which are often constructed as "untouched environments" and therefore subject to a range of legislative requirements. Strang's work challenges contemporary management practice which tends to view the environment as an objective (scientific) reality which has "natural" and "cultural" values. As Strang points out, "value" is embedded within the cultural construction. Furthermore, there is a perception that "natural resources" (at least) can be objectively quantified and on this basis, given value. This quantification of the environment is based on scientific values that are, of course, drawn from a particular cultural construction. In addition, there has been a tendency to conflate Aboriginal values with those of environmentalists without recognition that although there may well be some common ground, they are usually drawn from different cultural constructions.

In her description and analysis of the pastoralist group in the Mitchell River catchment, Strang makes the distinction between those who have had long-term associations with the region and those that are employees of absentee landowners. The latter are characterized as highly mobile, staying in the region for relatively short periods and having a tendency to be focused on the primary economic activity, i.e. grazing cattle: 
"Thus the land is a stage for human activities, rather than a medium of organisation, and the available roles could be performed interchangeably in any similar economic or social situation"(p. 280).

In Strang's analysis, the short-term, transient pastoralists appear to outnumber those who have long-term ties to the land. Again, this is an important point in terms of the debate surrounding Native Title. For many nonindigenous Australians, the Native Title debate is about differing relations to land. Whereas some nonindigenous interests in land (e.g., mining) can be characterized as overtly economic, there is a perception that those of pastoralists are less clearly economic and tempered by their relationship with land. As one of the long-term pastoralists noted:

People that own the land, and that have been on the land a long time, they've got a very strong attachment to it, just the same as the Aboriginals. And you can get the other sort of people that just buy it and do a few things to it and sell it - flog a few cattle off it...just to make a quid out of it. The genuine graziers...they look after it so that they can get the benefits out of it for years to come (Colin Hughes cited in V. Strang 1997: 206).

Although I am not suggesting that the long-term pastoralists have the same attachment to land as Aboriginal people, there is clearly a difference within the pastoral group. Of particular importance in terms of the public debate surrounding Native Title is the fact that within this area, the majority of pastoralists do not have long-term ties with the land.

One of the difficulties I have with Strang's work is that she does not make enough of these differences within the pastoralists' group. She often cites a particular long-term pastoralist whose values express a deeper relationship with the land that goes beyond economic function. For example:

I'd like to be buried here...I'll get up on the ridge there...When I go they can plant me there - they'll probably push me in a breakaway and let the crows eat me, you know!...I don't know if they need bother with a gravestone - just plant a tree on me or something...I like it here, and it's where I'm living, so there's a possibility I might like it here when I'm dead! (David Hughes cited in V. Strang 1997: 274).

Although she does draw a distinction between long- and short-term pastoralists, her conclusions regarding 'values' are more related to the latter group. I feel that the differences within the pastoralists' group could have been drawn more finely and wonder what effect this might have had on the conclusions which, as the title suggests relate to the 'uncommon ground' between the two groups.

Related to this, the analysis of Aboriginal constructions of environment and landscape is developed on the basis of a considerable amount of ethnographic examination. The Aboriginal people of west coast Cape York have had, as Strang herself points out, a long history of anthropological investigation, which began in the 1920s and 1930s, and was followed by a number of doctoral studies in the 1970s and 1980s. There is a rich body of ethnographic information that backgrounds Strang's own study and from which a detailed picture of the relationship between people, cosmology, and land can be drawn. In contrast, there has been little work of a similar nature (apart from Strang's) undertaken amongst the pastoralists. Given this, the comparison between the two groups is somewhat problematic. For example, Strang refers to differences in the way in which Aboriginal people and pastoralists have named the country. On the one hand, there is a wealth of information related to the way in which Aboriginal people name their country, and place names 
“...refer to related ancestral stories, and thus to the clans who own that country" (p. 219). In contrast, the meaning and stories behind European names (which obviously could paint a picture of European relationships to land, e.g. "Labour-in-Vain Yard, Mistake Dam and Broken Dray Creek...Dinner camp, Monday's Yard...Revolver Dam, Whisky Lagoon” (p. 218) are lost. Strang states that the loss of this information is at least partly related to the mobility of the Europeans in this region. It seems possible however that much of the information that is deemed to be lost might resurface if the pastoralist group was subjected to a similarly long history of investigation. It may be that the apparent absence of European relationships that go beyond economic function is rather related to current access to this information.

In the section that contrasts Aboriginal cosmology with that of the pastoralists, Strang refers to overarching frameworks such as Christianity, Science, Rationalism, etc. to explain the pastoralists' perspective. I do not deny that these underlie the perspectives of the pastoralists, however a more detailed explanation of the way in which these are located and played out amongst the pastoralist groups would have presented a more informed picture. There is a shift in the scale of the analysis which may be related to the degree to which we know and understand each group. This raises another issue, the extent to which "understanding" is assumed when there is a perception of "common ground" between researcher and those researched (i.e., Strang and the pastoralists). Strang does not appear to have been completely successful in disentangling notions of "self" and "other" in relation to the pastoralist group.

In spite of these reservations, I believe that Strang's work is an important pioneering study. She has identified that Aboriginal and non-Aboriginal people in Cape York have different similtaneous constructions of the environment in which they live and operate. Her study addresses issues that underlie Native Title, which is one of the most significant political and social debates that has engaged Australians this century. One of the major contributions of Strang's work is that she has reconstructed the comparative study within a frame of contemporary social issues.

\section{Reference Cited:}

\section{Greer, S.}

1995.The Accidental Heritage: archaeology and identity in northern Cape York. PhD thesis, Department of Anthropology and Archaeology, James Cook University, Townsville, Australia. 